\title{
Aplicação de Simulação Monte Carlo na Análise dos Impactos da Geração Distribuída Sobre um Alimentador Radial
}

\author{
Henrique P. Corrêa ${ }^{1}$, Flávio Henrique T. Vieira ${ }^{1}$, Marcelo S. de Castro ${ }^{1}$ \\ ${ }^{1}$ Escola de Engenharia Elétrica, Mecânica e de Computação, UFG, Goiânia 74605-010, Brazil \\ pires_correa@ufg.br, flavio_vieira@ufg.br, mcastro@ufg.br
}

\begin{abstract}
This work presents a case study concerning the operation of a distribution feeder with associated distributed generation, with the objective of discussing the importance of probabilistic modeling in the analysis of power systems with distributed generation. The impact over voltage profile is analyzed probabilistically by means of the Monte Carlo method, with multiple scenarios of feeder length and loading being considered. The results reinforce the fact that, even for simple systems, the stochastic character of distributed generation may not be ignored without incurrence in severe modeling errors.
\end{abstract}

Resumo. Este trabalho apresenta um estudo de caso relativo à operação de um alimentador de distribuição com geração distribuída, visando-se promover uma discussão sobre a importância da modelagem probabilística na análise de sistemas de potência com geração distribuída. O impacto sobre o perfil de tensão é analisado probabilisticamente via método Monte Carlo, considerando-se diferentes cenários de comprimento e carregamento do alimentador. Os resultados reforçam que, mesmo em sistemas simples, o caráter estocástico da geração distribuída não pode ser ignorado, sob pena de erros severos na modelagem.

\section{Introdução}

A crescente conscientização de consumidores e concessionárias de distribuição com relação à preservação ambiental por meio da utilização de energia renovável, aliada ao aumento de eficiência das tecnologias de geração fotovoltaica e à regulamentação da geração distribuída via resolução ANEEL no 482 [ANEEL b], vem provocando aumento ininterrupto na quantidade de unidades de geração fotovoltaica distribuída instaladas no país. Esta tendência pôde de fato ser comprovada em 2018, quando foi informado pela ANEEL que o número de conexões havia ultrapassado o montante de vinte mil unidades geradoras [ANEEL a]. Dentre estas, a modalidade fotovoltaica representou aproximadamente dois terços das adesões, o que reforça a relevância desta tecnologia no cenário nacional.

O aumento da potência de geração instalada pode ensejar múltiplos benefícios operacionais para as concessionárias de distribuição, dentre os quais encontram-se: melhoria dos níveis de tensão, alívio no carregamento dos alimentadores de distribuição e postergação de interrupções decorrentes de investimentos em reforços dos alimentadores [Daly and Morrison 2001]. Todavia, é amplamente reconhecido na literatura técnica que o excesso de geração distribuída instalada é capaz de causar problemas operacionais graves. Dentre estes, os mais frequentemente destacados na literatura são: aumento excessivo da tensão eficaz, níveis inadmissíveis de distorção harmônica e falhas na proteção 
em decorrência de reversões no fluxo de potência [Gomez et al. 2013]. Logo, a instalação de unidades geradoras não pode ser indiscriminada.

Nesse sentido, é necessário o estabelecimento de técnicas computacionais que permitam estimar os impactos na qualidade de energia em função da potência total instalada, permitindo-se que concessionárias obtenham estimativas quando da análise da geração fotovoltaica distribuída presente em seus sistemas. Os principais fatores que dificultam a estimação dos impactos como função da potência instalada são a intermitência da fonte solar e a aleatoriedade de alocação da potência total ao longo do sistema. O primeiro fator encontra-se associado às rápidas variações de irradiância que ocorrem ao longo do dia, da qual a potência gerada por painéis fotovoltaicos é, aproximadamente, linearmente dependente. O segundo, por outro lado, deve-se ao fato da potência total encontrar-se distribuída entre muitas unidades geradoras de diferentes potências, para as quais existe um número muito elevado de possíveis locais de instalação ao longo da rede.

Pela discussão prévia, o aspecto estocástico da geração fotovoltaica distribuída não recomenda a utilização de métodos determinísticos para a análise de sua influência sobre a qualidade da energia. Logo, é necessária a aplicação de métodos computacionais que contemplem as múltiplas instâncias possíveis de geração e, a partir destas, possam prover estimativas probabilísticas dos parâmetros de interesse, dentre os quais é destacado o perfil de tensão no presente trabalho. No que segue, é apresentado um estudo de caso com o objetivo de exemplificar como o método Monte Carlo pode ser aplicado pelas concessionárias neste contexto. O estudo é parametrizado em função de comprimento e carregamento do alimentador, permitindo-se visualizar a influência destes na rede.

\section{Fonte dos Dados Utilizados - Projeto $P \& D 364$}

É notável a tendência de aumento nos estímulos a investimentos, tanto operacionais quanto da perspectiva de pesquisa e desenvolvimento, que visam enquadrar as redes de distribuição no conceito de redes inteligentes. Dentre as principais mudanças tidas como caracterizadoras deste enquadramento, encontram-se a implementação de soluções de telemetria para monitoramento individual de energia e potência dos consumidores e a integração plena de tecnologias de geração distribuída conectadas à rede.

Nesse contexto, o projeto P\&D 364 - Projeto 51 Telhados em Nova Veneza - GO: Sistema Solar Fotovoltaico de Microgeração Distribuída Conectada à Rede de Baixa Tensão da Celg-D em 51 UCs Dentro do Conceito de Redes Elétricas Inteligentes, conduzido pela Universidade Federal de Goiás em parceria com a Enel Distribuição Goiás, foi elaborado com o objetivo de se implementar e estudar a operação de uma estrutura de rede inteligente contemplando 51 unidades consumidoras do município de Nova Veneza. Um dos principais focos do projeto foi implantar usinas fotovoltaicas, sobre telhados, em unidades estaduais de ensino situadas em diferentes cidades do estado de Goiás. Como ilustração dos resultados alcançados, são exibidas, na Figura 1, fotografias de módulos fotovoltaicos instalados em duas das unidades consumidoras envolvidas no projeto.

No projeto, a seleção das unidades consumidoras para instalação dos painéis foi balizada por critérios socioeconômicos. Todavia, é reconhecido que a avaliação dos impactos da geração sobre a qualidade da energia é de grande importância. Nesse sentido, as simulações deste trabalho visaram, no âmbito do $P \& D 364$, utilizar dados dos painéis e alimentadores para avaliar os impactos sobre o perfil de tensão em decorrência da geração. 


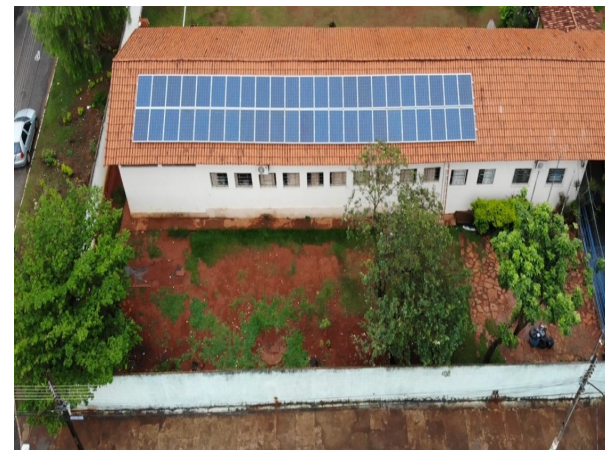

(a) Colégio José Peixoto

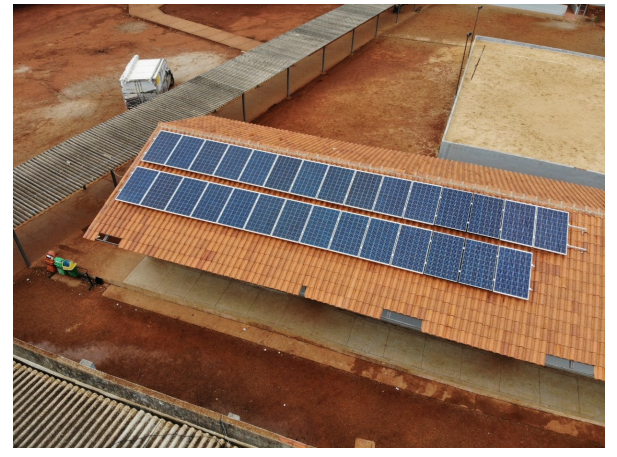

(b) Colégio Manoel Vila Verde

Figura 1. Sistemas fotovoltaicos instalados no âmbito do $P \& D 364$

\section{Simulação Monte Carlo}

O método Monte Carlo consiste em gerar aleatoriamente um número elevado $S$ de instâncias para simulação, cujos resultados são armazenados e subsequentemente utilizados na obtenção das funções densidade de probabilidade relativas às variáveis de interesse. No presente estudo, as variáveis visadas são as magnitudes de tensão nas barras do alimentador, doravante designadas por $V_{i}, \operatorname{com} i=1,2, \ldots, N$, em que $N$ é o número total de barras. Anteriormente à execução das $S$ iterações, os fatores estocásticos a serem considerados (irradiância e alocação de geradores distribuídos) devem ser modelados por meio de funções massa de probabilidade [Robert and Casella 2010], as quais serão especificadas na próxima seção.

Seja uma variável aleatória discreta $X$ com função massa de probabilidade $f_{X}(x)$ e domínio $\mathcal{X}=\left\{x_{1}, x_{2}, \ldots, x_{K}\right\}$, com probabilidades $P\left(x_{i}\right)=p_{i}, K \in \mathbb{N}^{*}$, associada a qualquer um dos parâmetros aleatórios. Para realizar a amostragem de $X$ e obterse as amostras $x_{s}$, com $s=1,2, \ldots, S$, utiliza-se o método da transformada inversa [Robert and Casella 2010, Abdelaziz 2017], o qual é descrito a seguir. Dada uma variável aleatória contínua distribuída uniformemente $U(0,1)$, gera-se aleatoriamente um número $0<u<1$. Verifica-se, então, para qual $l$ as seguintes desigualdades são satisfeitas:

$$
F_{X}\left(x_{l-1}\right)=\sum_{i=1}^{l-1} p_{i}<u \leq \sum_{i=1}^{l} p_{i}=F\left(x_{l}\right)
$$

onde $F_{X}(x)$ é a distribuição cumulativa de $X$. Dado $l$ para o qual (1) é satisfeita, a amostra aleatória $X=x_{l}$ é selecionada. Após este procedimento ser repetido para todas os parâmetros aleatórios considerados (neste caso, irradiância e alocação de unidades geradoras) e as correspondentes amostras serem obtidas, estas são utilizadas como entradas para simulação do sistema estudado (em outros termos, solução do fluxo de carga da rede). As variáveis de interesse obtidas via simulação são armazenadas e o procedimento descrito é repetido até que sejam realizadas $S$ iterações.

Sejam as amostras conjuntas dos parâmetros estocásticos denotadas por $s_{j}, j=$ $1,2, \ldots, S$. A saída do método Monte Carlo consiste em magnitudes de tensão nas barras do sistema $V_{i}^{n}\left(s_{j}\right), i=1,2, \ldots, N$, para cada amostra $s_{j}$. Os $S$ valores de $V_{i}\left(s_{j}\right)$ podem ser utilizados para estimar a distribuição da magnitude de tensão na barra $i$, ou seja, $f_{V_{i}}(v)$, 
$v \in \mathbb{R}^{+}$. Para isso, um valor de incremento $\Delta V$ deve ser escolhido para particionar o intervalo $\left(0, \max _{j} V_{i}\left(s_{j}\right)\right]$ em subintervalos disjuntos:

$$
\left(0, \max _{j} V_{i}\left(s_{j}\right)\right]=\bigcup_{r=1}^{L}((r-1) \Delta V, r \Delta V]
$$

onde $L=\frac{\max _{j} V_{i}\left(s_{j}\right)}{\Delta V}$. Para os subintervalos obtidos, a distribuição discreta estimada $\hat{f}_{V_{i}}(r)$, $r=1,2, \ldots, L$, pode ser computada via frequências relativas:

$$
\hat{f}_{V_{i}}(r)=\frac{\#\left\{V_{i}\left(s_{j}\right) \mid(r-1) \Delta V<V_{i}\left(s_{j}\right) \leq r \Delta V\right\}}{S}
$$

em que o símbolo \# denota o número de elementos de um conjunto. Para cada subintervalo, a média dos extremos é considerada como o valor representativo. Logo, para o subintervalo $((r-1) \Delta V, r \Delta V]$, este valor é igual a $\frac{2 r-1}{2} \Delta V$ e, portanto:

$$
\hat{f}_{V_{i}}(r)=\hat{P}\left\{V_{i}\left(s_{j}\right)=\frac{2 r-1}{2} \Delta V\right\}
$$

Finalmente, após a execução dos $S$ fluxos de carga, as distribuições $\hat{f}_{V_{i}}(r)$ são obtidas para todos $i=1,2, \ldots, N$.

\section{Modelagem do Alimentador e Fator de Penetração}

Para a modelagem do alimentador radial e unidades geradoras distribuídas, foram utilizados dados reais obtidos no âmbito do projeto $P \& D$ 364. Dentre os dados disponíveis no projeto, são utilizadas as seguintes especificações de alimentador e módulo fotovoltaico, referentes à instalação executada na escola estadual José Peixoto, em Nova Veneza, para efeito de modelagem do sistema considerado:

- Alimentador radial e trifásico com impedância $2,1288+j 2,1972 \Omega / \mathrm{km}$, suprido por transformador $13,8 \mathrm{kV} / 380 \mathrm{~V}$ de potência nominal $75 \mathrm{kVA}$.

- Módulo fotovoltaico CS6P-265P, de potência 265W [CanadianSolar ].

Com o objetivo de se contemplarem múltiplos cenários de comprimento e carregamento do alimentador, foram arbitrados os seguintes valores a serem simulados em todas as suas combinações comprimento/carregamento possíveis:

- Comprimentos de alimentador $(L): 100 \mathrm{~m}$ e $300 \mathrm{~m}$.

- Carregamento do alimentador $\left(P_{c}\right)$ : 25kVA e $75 \mathrm{kVA}$.

Em particular, o valor de carregamento 25kVA foi selecionado com o objetivo de contemplar-se a possibilidade de ocorrer excesso de geração, ou seja, a combinação de intensa geração fotovoltaica distribuída com baixa demanda ao longo do alimentador.

Assume-se que a carga é equilibrada entre as fases e uniformemente distribuída em três parcelas ao longo do alimentador. A modelagem adotada para o alimentador assume impedâncias idênticas por fase e um sistema a três fios (sem condutor neutro). Ademais, 


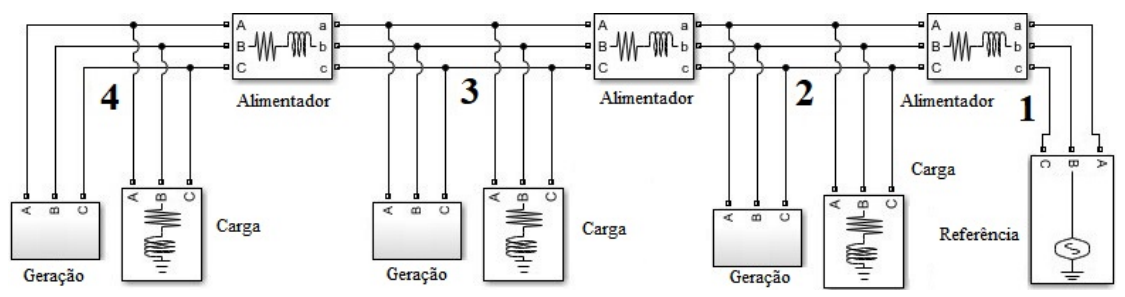

Figura 2. llustração da modelagem considerada para o alimentador

os terminais de baixa tensão do transformador são modelados por um barramento infinito. Logo, o sistema pode ser representado por um circuito com $N=4$ nós: um nó de referência e três nós de carga igualmente espaçados, como ilustrado na Figura 2.

Finalmente, para cada uma das quatro combinações de comprimento e carregamento do alimentador, são consideradas múltiplas instâncias do nível de penetração da geração distribuída. Para quantificar a geração instalada e, por consequência, o grau de penetração, foi utilizada a seguinte definição de fator de penetração:

$$
F P=\frac{P_{\text {fotovoltaica }}(\mathrm{kW})}{S_{\text {transformador }}(\mathrm{kVA})}
$$

em que $P_{\text {fotovoltaica }}$ é a potência fotovoltaica total instalada e $S_{\text {transformador }}$ é a potência nominal do transformador que supre o alimentador. Para cada combinação comprimento/carregamento, foram simulados os casos $F P \in\{0,25+0,25 \cdot i ; i=1,2, \ldots, 5\}$. Os passos listados a seguir sumarizam o procedimento de simulação adotado:

1. Especifique o comprimento e carregamento do alimentador;

2. Especifique o fator de penetração;

3. Dado o fator de penetração, aloque aleatoriamente painéis fotovoltaicos nas barras até que se alcance a correspondente potência instalada;

4. Por meio da distribuição de irradiância, gere aleatoriamente a porcentagem da potência nominal que será injetada pelos painéis;

5. Repita as etapas 3 e 4 até que sejam completadas $S$ iterações do método Monte Carlo, obtendo as densidades de probabilidade das tensões nas barras;

6. Retorne à etapa 1 até que sejam exauridas todas as combinações $L / P_{c} / F P$.

A título de simplificação, foi assumido que a irradiância solar obedece a uma distribuição uniforme. Nesse sentido, a potência ativa gerada em cada barra pode ser modelada diretamente, de acordo com a seguinte equação:

$$
P_{G, i}=\left[P_{G, i}\right]_{o} \cdot \frac{U^{\prime}(0,100)}{100}
$$

em que $U^{\prime}(0,100)$ é a distribuição uniforme discreta e $\left[P_{G, i}\right]_{o}$ é a potência nominal alocada na barra $i$. Logo, a potência injetada pode variar de $0 \%$ a $100 \%$ da potência instalada, em incrementos de $1 \%$. Supõe-se que, em (6), tem-se $i=2,3,4$, ou seja, não há alocação de geração distribuída na barra de referência. Por fim, note-se que é assumida geração em fator de potência unitário, ou seja, sem injeção ou consumo de potência reativa. 
Para a alocação de cada painel fotovoltaico, assume-se igual probabilidade para as três barras do sistema. Logo, quando da alocação de um painel, uma amostra da seguinte variável é gerada, cujo valor define a barra em que será feita a alocação:

$$
X_{a l o c}=U^{\prime}(2,4)
$$

em que há, portanto, probabilidade igual a ${ }^{1 / 3}$ de que cada painel seja alocado em qualquer uma das barras de carga. Por fim, definida a barra em que o painel será alocado, uma variável aleatória análoga é utilizada para determinar a fase em que este será conectado:

$$
X_{\text {fase }}=U^{\prime}(1,3)
$$

onde os valores $X_{\text {fase }}=1,2,3$ correspondem à fases $A, B$ e $C$, respectivamente.

\section{Resultados de Simulação}

Nesta seção, são apresentados os resultados obtidos por meio das simulações Monte Carlo. Para cada valor de FP considerado, o número de iterações foi $S=3000$ e o incremento selecionado para a tensão foi $\Delta V=0,001 \mathrm{~V}$. Os resultados são apresentados nas Figuras 3 a 6, na forma das densidades de probabilidade estimadas para as magnitudes de tensão. Por concisão, apenas os resultados da fase $A$ são apresentados.

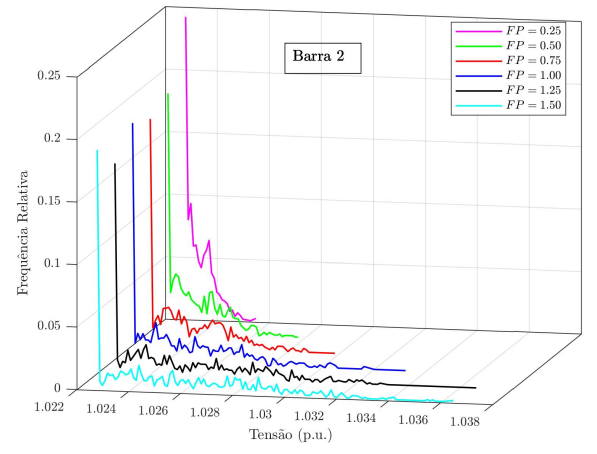

(a) Barra 2

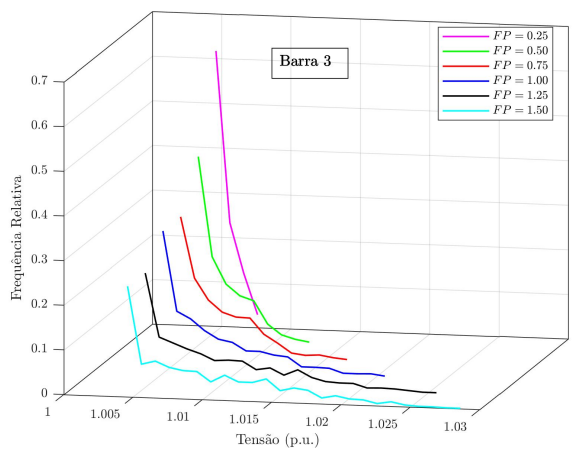

(b) Barra 3

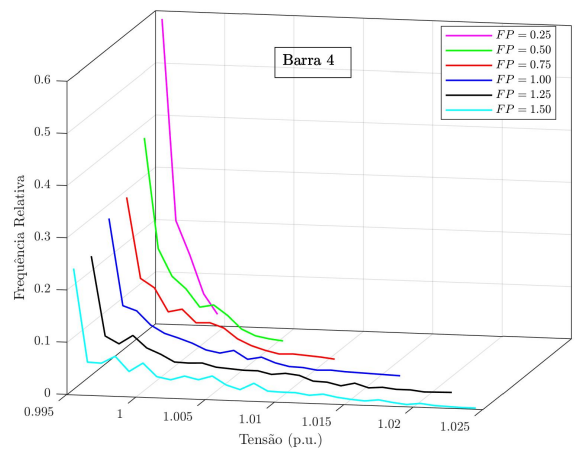

(c) Barra 4

Figura 3. Resultados da simulação Monte Carlo com $L=100 \mathrm{~m}$ e $P_{c}=75 \mathrm{kVA}$ 


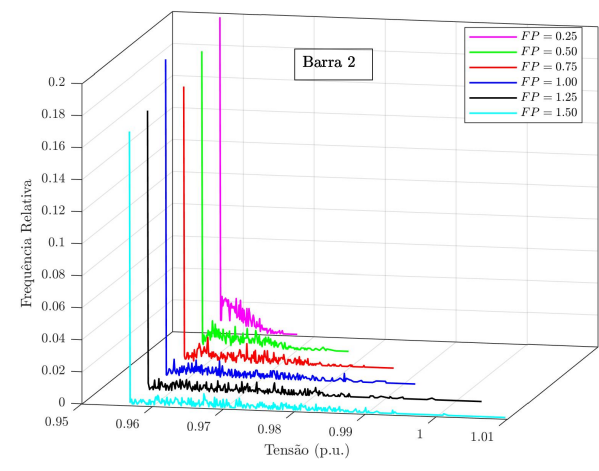

(a) Barra 2

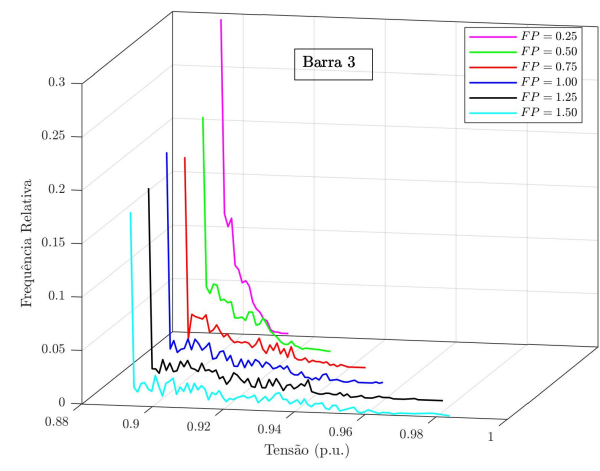

(b) Barra 3

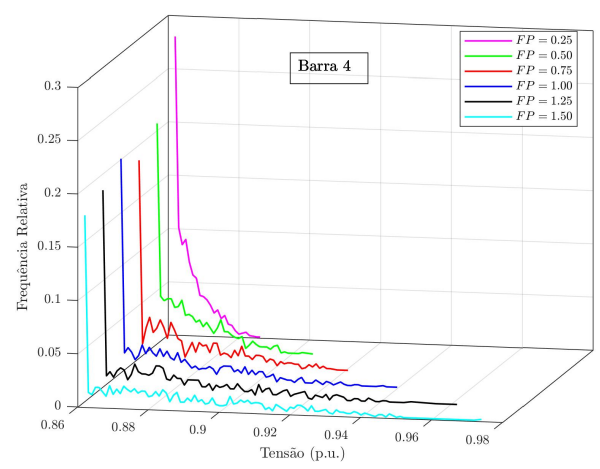

(c) Barra 4

Figura 4. Resultados da simulação Monte Carlo com $L=300 \mathbf{m}$ e $P_{c}=75$ kVA

Nos itens elencados abaixo, são apresentados e discutidos os aspectos mais relevantes que puderam ser observados nos resultados obtidos.

- O principal efeito surtido pelo aumento do carregamento do alimentador é o deslocamento das distribuições de probabilidade para maiores valores no eixo das magnitudes de tensão; por outro lado, as variâncias das distribuições para um mesmo fator de penetração tendem a permanecer similares. Ou seja, a diminuição do carregamento de alimentador tende a produzir maiores valores de tensão, embora a dispersão destes permaneça aproximadamente constante.

- O comprimento $L$ influi sobre dois aspectos relevantes: maiores comprimentos tendem a simultaneamente aumentar a dispersão da distribuição e deslocá-la para menores valores no eixo das tensões. O primeiro efeito advém do aumento de impedância entre os pontos de geração, o que implica que as diferentes configurações de alocação da geração passam a ter efeitos mais significativos sobre o perfil de tensão, aumentando a diversidade dos valores de tensão obtidos. O deslocamento das distribuições para a esquerda deve-se às maiores quedas de tensão em função de maior comprimento do alimentador; nesse sentido, a vizinhança da distribuição de tensões é determinada de maneira análoga àquela exposta no item anterior.

- Em circustâncias de carregamento nominal, especialmente para comprimentos maiores de alimentador, verifica-se que a geração distribuída propicia melhoria nos perfis de tensão, não apresentando riscos significativos de sobretensão nas bar- 


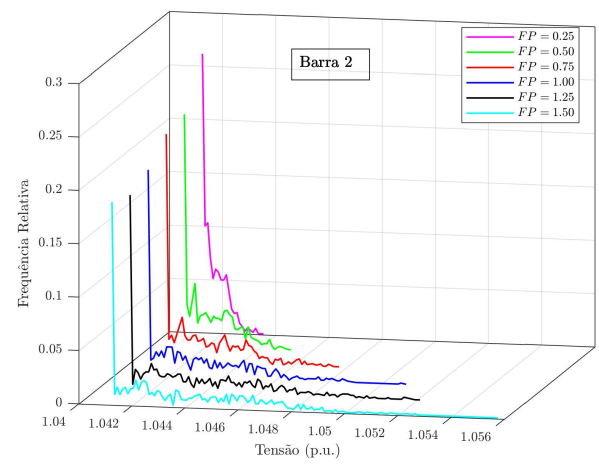

(a) Barra 2

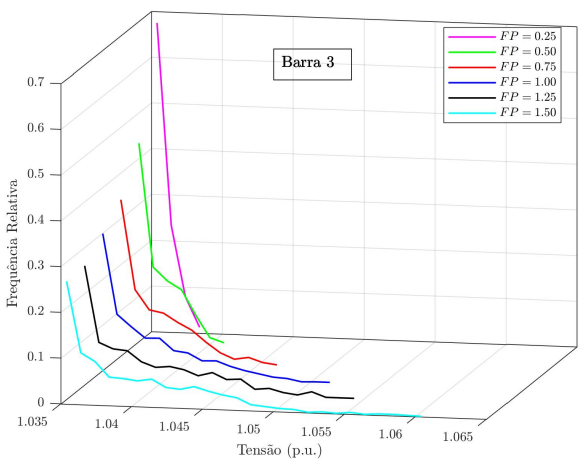

(b) Barra 3

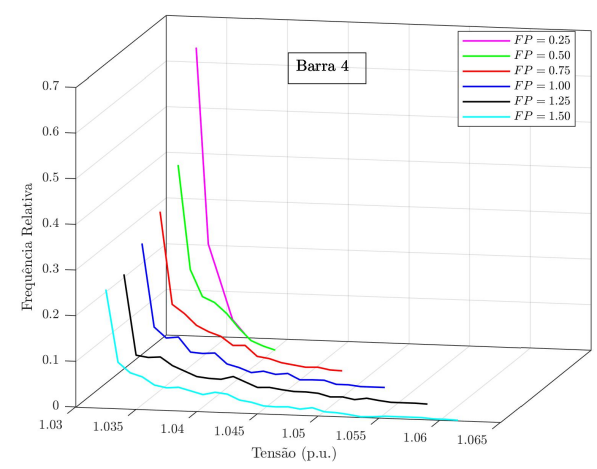

(c) Barra 4

Figura 5. Resultados da simulação Monte Carlo com $L=100 \mathbf{m}$ e $P_{c}=25$ kVA

ras inclusive para elevados fatores de penetração. Isso se justificava pelo fato de carregamentos relativamente elevados tenderem a conduzir as tensões do sistema para valores abaixo de 1 p.u., o que é compensado pelo alívio de carga propiciado pela instalação dos painéis fotovoltaicos.

- A posição de uma barra, com relação à barra de referência, influencia acentuadamente sua respectiva distribuição de tensão. Os resultados demonstram que, quanto mais próxima da barra de referência se encontra uma determinada barra, menos dispersa tende a ser sua distribuição de tensões. Isso pode ser explicado pelo fato de que os menores níveis de impedância entre a origem do sistema e barras próximas desta fazem com que as tensões destas sejam mais fortemente vinculadas à tensão da referência, independentemente de geração ou carregamento.

- Os resultados confirmam a asserção de que não é suficiente a análise determinística dos impactos da geração distribuída sobre alimentadores de distribuição. De fato, nos casos simulados em que houve maior reincidência de um mesmo valor de tensão em uma das barras, a frequência relativa permaneceu próxima a 70\%. Logo, a dispersão dos valores de tensões como função da aleatoriedade de geração não pode ser negligenciada em uma análise precisa da geração distribuída.

- De modo geral, os resultados demonstram que a probabilidade de ocorrência de sobretensões é aumentada para alimentadores com carregamento leve e alta penetração de geração distribuída. Por outro lado, a geração distribuída traz efei- 


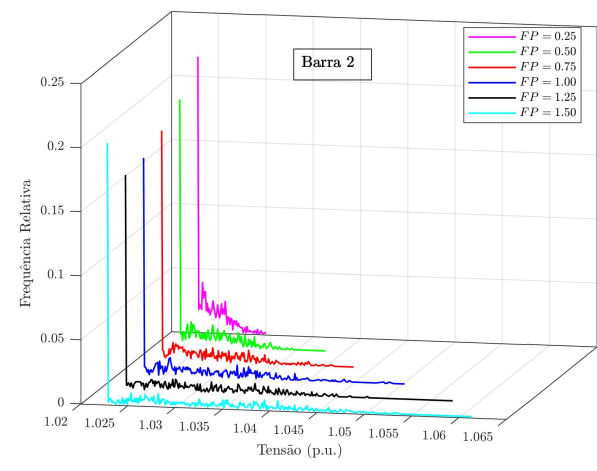

(a) Barra 2

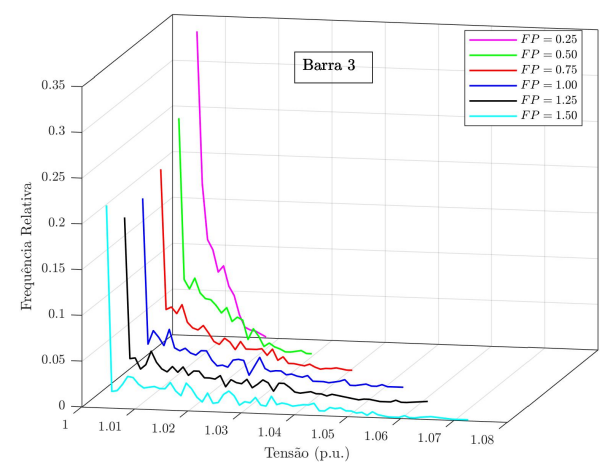

(b) Barra 3

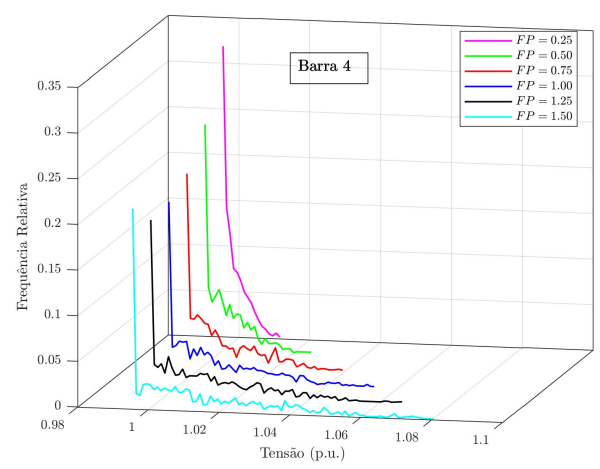

(c) Barra 4

Figura 6. Resultados da simulação Monte Carlo com $L=300 \mathbf{m}$ e $P_{c}=25$ kVA

tos benéficos para alimentadores longos com carga elevada, nos quais o risco de sobretensão não se torna significativo mesmo para elevados fatores de penetração.

\section{Considerações Adicionais}

No estudo de caso analisado neste trabalho, algumas simplificações foram utilizadas na modelagem do alimentador de distribuição, as quais consistem na suposição de: cargas equilibradas, impedâncias idênticas em todas as fases dos trechos de alimentador e sistema a três condutores (ou seja, sem a presença do condutor neutro). Os resultados indicam que foi possível, mesmo utilizando-se destas simplificações, evidenciar os efeitos do comportamento estocástico da geração sobre as tensões nas barras do alimentador.

Todavia, uma análise mais precisa dos sistemas de distribuição requer a modelagem de todos os fatores que foram desconsiderados por meio das suposições acima mencionadas. De fato, sabe-se que as cargas tendem a ser significativamente desequilibradas na distribuição, principalmente devido à abundância de ramais monofásicos e bifásicos derivados dos alimentadores principais [Kim 2018]. Ademais, em decorrência das linhas de distribuição serem dispostas assimetricamente e não serem transpostas ao longo de suas extensões, pode ocorrer desequilíbrio considerável entre as impedâncias mútuas e próprias das fases [Liu and Milanovic 2015, Yan and Saha 2013]. Por fim, o condutor neutro é utilizado na maior parte dos sistemas de distribuição em baixa tensão, sendo sua incorporação aos modelos de alimentador necessária em análises detalhadas. 
Todavia, modelar explicitamente o condutor neutro e estudar o comportamento das tensões em seus nós em função das impedâncias de aterramento e da geração distribuída pode ser oneroso computacionalmente. De fato, uma simulação de fluxo de carga seria necessária para cada possível combinação de valores para as impedâncias de aterramento. Unindo este requisito às múltiplas iterações do método Monte Carlo, o tempo de processamento pode tornar-se inaceitável. Em trabalhos futuros, pretende-se desenvolver métodos para atenuar a complexidade computacional associada a este tipo de simulação.

\section{Conclusão}

Neste trabalho, foi apresentado um estudo de caso baseado em dados do $P \& D 364$ em que foram analisados, via simulação Monte Carlo, os impactos da geração fotovoltaica distribuída sobre as magnitudes de tensão em um alimentador radial. O objetivo do estudo foi demonstrar a importância de se considerar fatores estocásticos na modelagem da geração distribuída. Os resultados obtidos demonstraram que as variações de tensão no alimentador, decorrentes da aleatoriedade inerente à geração distribuída, não podem ser ignoradas sem incidência em erros significativos de modelagem.

\section{Agradecimentos}

Este trabalho foi desenvolvido dentro das pesquisas realizadas no Projeto de P\&D número PD-06072-0364/2015 da Aneel, financiado pela Celg Distribuidora.

\section{Referências}

Abdelaziz, M. (2017). Gpu-opencl accelerated probabilistic power flow analysis using monte-carlo simulation. Electric Power Systems Research, 147:70 - 72.

ANEEL. 'Geração distribuída ultrapassa 20 mil conexões' (2018). Assesoria de Imprensa da Agência Nacional de Energia Elétrica.

ANEEL. Resolução Normativa no 482 de 2012. Agência Nacional de Energia Elétrica.

CanadianSolar. Datasheet do módulo CS6P-260/265/270P. CanadianSolar.

Daly, P. A. and Morrison, J. (2001). Understanding the potential benefits of distributed generation on power delivery systems. In 2001 Rural Electric Power Conference. Papers Presented at the 45th Annual Conference (Cat. No.01CH37214), pages A2/1-A213.

Gomez, J. C., Vaschetti, J., Coyos, C., and Ibarlucea, C. (2013). Distributed generation: impact on protections and power quality. IEEE Latin America Transactions, 11(1):460-465.

Kim, Y. (2018). Development and analysis of a sensitivity matrix of a three-phase voltage unbalance factor. IEEE Transactions on Power Systems, 33(3):3192-3195.

Liu, Z. and Milanovic, J. V. (2015). Probabilistic estimation of voltage unbalance in mv distribution networks with unbalanced load. IEEE Transactions on Power Delivery, 30(2):693-703.

Robert, C. and Casella, G. (2010). Introducing Monte Carlo Methods with R. Springer.

Yan, R. and Saha, T. K. (2013). Investigation of voltage imbalance due to distribution network unbalanced line configurations and load levels. IEEE Transactions on Power Systems, 28(2):1829-1838. 